\title{
MicroRNA-23b-3p targets non-SMC condensing I complex subunit $G$ to promote proliferation and inhibit apoptosis of colorectal cancer cells via regulation of the PI3K/AKT signaling pathway
}

\author{
PEIDONG LI ${ }^{1,2}$, JING WEN $^{1,2^{*}}$, XIRU REN $^{3^{*}}$, YU ZHOU $^{1,2}$, YUAN XUE $^{1,2}$, \\ ZAIHUA YAN $^{1,2}$, SHENGJIE LI ${ }^{1,2}$, HONGPENG TIAN ${ }^{1}$, \\ XUE-GUI TANG ${ }^{4}$ and GUANG-JUN ZHANG ${ }^{1,2}$
}

\author{
${ }^{1}$ The Second Department of Gastrointestinal Surgery, The Affiliated Hospital of North Sichuan Medical College; \\ ${ }^{2}$ Institute of Hepatobiliary, Pancreatic and Intestinal Disease, North Sichuan Medical College; \\ ${ }^{3}$ Nanchong Inspection Institute for Food and Drugs; ${ }^{4}$ Anorectal Department of \\ Integrated Traditional Chinese and Western Medicine, The Affiliated Hospital of North Sichuan Medical College, \\ Nanchong, Sichuan 637000, P.R. China
}

Received June 16, 2021; Accepted August 31, 2021

DOI: $10.3892 / \mathrm{ol} .2021 .13073$

\begin{abstract}
Colorectal cancer (CRC) is one of the most common types of malignancy worldwide and has a poor prognosis. Non-SMC condensing I complex subunit G (NCAPG) has been reported to be upregulated in numerous types of malignant tumor. However, to the best of our knowledge, its clinicopathological and biological significance in CRC remain to be elucidated. The results of the present study revealed that NCAPG expression levels were upregulated in human CRC tissues and cell lines. The upregulated expression of NCAPG was positively associated with patient clinicopathological characteristics, such as differentiation and tumor size, and independently associated with poor survival. Consistent with the clinical observations, NCAPG was discovered to promote the proliferation and inhibit the apoptosis of CRC cells. Moreover, NCAPG-knockdown inhibited CRC cell proliferation by regulating the PI3K/AKT
\end{abstract}

Correspondence to: Dr Xue-Gui Tang, Anorectal Department of Integrated Traditional Chinese and Western Medicine, The Affiliated Hospital of North Sichuan Medical College, 1 Maoyuan South Road, Nanchong, Sichuan 637000, P.R. China

E-mail:txg668nc@sohu.com

Dr Guang-Jun Zhang, The Second Department of Gastrointestinal Surgery, The Affiliated Hospital of North Sichuan Medical College, 1 Maoyuan South Road, Nanchong, Sichuan 637000, P.R. China

E-mail: zhanggj1977@126.com

*Contributed equally

Key words: colorectal cancer, non-SMC condensing I complex subunit G, microRNA-23b-3p, proliferation, apoptosis, PI3K/AKT pathway signaling pathway. Furthermore, NCAPG was identified as a potential target of microRNA (miR)-23b-3p, which was subsequently demonstrated to negatively regulate NCAPG expression. In conclusion, the findings of the current study indicated that the miR-23b-3p/NCAPG/PI3K/AKT signaling axis may play an important role in CRC carcinogenesis, and the status of the molecule may represent a promising prognostic marker for the disease.

\section{Introduction}

Colorectal cancer (CRC) is the third most common type of cancer worldwide, and both its morbidity and mortality rates continue to increase each year in China (1). However, despite significant advancements in the identification and development of novel diagnostic markers and therapeutic methods, the overall outcome of patients with CRC remains poor (2). Therefore, it is important to identify emerging predictive biomarkers, and the underlying mechanisms of action, to promote the development of more individualized treatment strategies for patients with CRC.

Non-SMC condensing I complex subunit G (NCAPG) is a protein involved in chromosome condensation, which is associated with mitosis (3). To exert its effects, human NCAPG activates condensin by regulating ATPase activity (4). Over the past few years, an accumulating number of studies have reported that NCAPG is involved in the pathogenesis of various types of cancer, including breast (5), gastric $(6,7)$ and prostate cancer (8), hepatocellular carcinoma (HCC) (9) and ovarian cancer (10). In our previous study, gene microarray analysis revealed that the expression levels of NCAPG were upregulated in CRC specimens (11). However, to the best of our knowledge, the biological functions and clinical implications of NCAPG in CRC remain to be determined. 
MicroRNAs (miRNAs/miRs), which are highly conserved small non-coding RNA molecules, primarily exert their functions at the post-transcriptional level by preventing mRNA translation via binding to the 3'-untranslated region (UTR) of downstream target mRNAs (12). Increasing evidence has suggested that miRNAs may be involved in the regulation of biological behaviors in a variety of cancer cell types (13), and multiple studies have reported that the expression levels of miR-23b-3p are downregulated in several types of cancer (14). In CRC, downregulated expression levels of miR-23b-3p were found be associated with 5-fluorouracil resistance (15). However, the exact mechanisms underlying the effects of NCAPG, and its association with miR-23b-3p in CRC, are poorly understood.

The present study aimed to determine the expression levels of NCAPG in CRC tissues and cell lines. Then, the clinical significance of NCAPG expression in CRC, and the effects of NCAPG on the proliferation and apoptosis of CRC cells, were investigated. Moreover, the study sought to determine the mechanisms underlying the actions of NCAPG by identifying the potential signaling pathway through which it exerts its effects in CRC.

\section{Materials and methods}

Patient samples. The patient experimental protocol was approved by the Ethics Committee of The Affiliated Hospital of North Sichuan Medical College (Nanchong, China) [approval no. 2021ER(A)005], and all patients provided written informed consent prior to study participation. CRC and adjacent non-carcinoma samples were obtained from 88 patients with CRC who underwent surgical resection at The Affiliated Hospital of North Sichuan Medical College between March 2012 and May 2014. After excision, the specimens were immediately frozen in liquid nitrogen and stored at $-80^{\circ} \mathrm{C}$. Among the 88 patients, 5 were excluded from the study after being diagnosed with liver or lung metastasis. The inclusion criteria were as follows: i) Patients were diagnosed with colorectal cancer through pathological examination for the first time; and ii) no distant tumor metastasis was detected. Exclusion criteria: i) Patients with other malignant tumors, hematological diseases, severe complications and/or immune system diseases; ii) patients treated with preoperative chemotherapy, radiotherapy or other therapeutic modalities; and iii) patients who were unwilling to participate in the present study. Detailed clinical characteristics of the enrolled patients are presented in Table SI.

Cell lines and culture. CRC cells (HCT116, HCT15, SW480, SW620 and LoVo), FHC cells (a human normal colon epithelium cell line) and 293T cells were purchased from the American Type Culture Collection. CRC cell lines were cultured in DMEM supplemented with $10 \%$ FBS (both Invitrogen; Thermo Fisher Scientific, Inc.). FHC and 293T cells were cultured in DMEM/F12 (Sigma-Aldrich; Merck KGaA) supplemented with $10 \%$ FBS. All cells were maintained at $37^{\circ} \mathrm{C}$ in a humidified incubator with $5 \% \mathrm{CO}_{2}$. For treatment with 740Y-P (PI3K/AKT signal activator), cells were seeded into 6 -well plates $\left(1 \times 10^{5}\right.$ cells/well) and incubated with $20 \mu \mathrm{M} 740 \mathrm{Y}-\mathrm{P}$ (MedChemExpress) for a $1 \mathrm{~h}$ at $37^{\circ} \mathrm{C}$.
Reverse transcription-quantitative (RT-q PCR). Total RNA was extracted from CRC tissues and cell lines using TRIzol ${ }^{\circledR}$ reagent (Invitrogen), according to the manufacturer's protocol. For the detection of NCAPG mRNA expression, reverse transcription was performed using the PrimeScript RT Reagent kit (Takara Bio, Inc.). qPCR was subsequently performed using SYBR Premix Ex Taq (Takara Biotechnology Co., Ltd.) on an ABI 7500 Real-Time PCR Detection system (Applied Biosystems; Thermo Fisher Scientific, Inc.). For the detection of miRNA expression, qPCR was performed using a TaqMan miRNA assay (Applied Biosystems; Thermo Fisher Scientific, Inc.) with miR-23b-3p specific primers (Applied Biosystems; Thermo Fisher Scientific, Inc.). The following thermocycling conditions were used for qPCR: $95^{\circ} \mathrm{C}$ for $10 \mathrm{~min}$; followed by 40 cycles of $95^{\circ} \mathrm{C}$ for $30 \mathrm{sec}, 60^{\circ} \mathrm{C}$ for $30 \mathrm{sec}$ and $72^{\circ} \mathrm{C}$ for $30 \mathrm{sec}$ and final extension at $72^{\circ} \mathrm{C}$ for $2 \mathrm{~min}$. The following primer sequences were used for qPCR: NCAPG forward, 5'-AAGTTA GACGGGCAGTGTTATC-3' and reverse, 5'-CAGCTTTCT GACAGCCTCTT-3'; miR-23b-3p, forward, 5'-ATCACATTG CCAGGGATTACCTTTTTT-3' and reverse, 5'-AATTAA AAAAGGTAATCCCTGGCAATGTGATGGCC-3'; GAPDH forward, 5'-TGACTTCAACAGCGACACCCA-3' and reverse, 5'-CACCCTGTTGCTGTAGCCAAA-3'; and U6 forward, 5'-CTCGCTTCGGCAGCACA-3' and reverse, 5'-AACGCT TCACGAATTTGCGT-3'. The expression levels were quantified using the $2^{-\triangle \Delta \mathrm{Cq}}$ method (16). GAPDH and U6 were used as the internal controls for the normalization of mRNA and miRNA expression, respectively.

Western blotting. Total protein was extracted from cells using complete cell lysis-M buffer (Roche Applied Science) supplemented with protease/phosphatase inhibitor cocktail (Cell Signaling Technology, Inc.). The proteins (40 $\mu \mathrm{g} / \mathrm{lane})$ were separated via SDS-PAGE, transferred onto PVDF membranes and blocked with $5 \%$ fat-free milk for $1.5 \mathrm{~h}$ at room temperature. The blocked membranes were subsequently incubated with the following primary antibodies overnight at $4^{\circ} \mathrm{C}$ : Anti-PI3K (cat. no. 4292; 1:1,000), anti-phosphorylated (p)-PI3K (cat. no. 17366; 1:1,000), anti-AKT (cat. no. 4685; 1:1,000), anti-p-AKT (cat. no. 13038; 1:1,000), anti-Bcl-2 (cat. no. 4233; 1:1,000), anti-Bax (cat. no. $2744 ; 1: 1,000)$ and anti-GAPDH (cat. no. 5174; 1:500) (all Cell Signaling Technology, Inc.), and anti-NCAPG (cat. no. ab251864; 1:750; Abcam). Following primary antibody incubation, the membranes were incubated with an HRP-conjugated secondary antibody (1:5,000; cat. no. ab7090; Abcam) for $1 \mathrm{~h}$ at $37^{\circ} \mathrm{C}$. Protein bands were visualized using ECL reagents (MilliporeSigma) and GAPDH was used as the internal loading control.

Cell transfection. To construct NCAPG-knockdown CRC cells, lentiviral short hairpin RNAs (shRNA/sh) targeting NCAPG were designed by Shanghai GeneChem Co.,Ltd. The sequences for the shRNAs were as follows: shNCAPG1 forward, 5'-GAT CCCGGGCAGTGTTATCATGTATTCTCGAGAATACAT GATAACACTGCCCGTTTTTT-3' and reverse, 5'-CTTAAA AAAAACGGGCAGTTTATCATGTATTCTCGAGAATAC ATGATA ACACTGCCCG-3'; and shNCAPG2 forward, 5'-GATCCGCCTTA ACAGTACATGACAATCTCGAGATT GTCATGTACTGTTAAGGCTTTTTT-3' and reverse, 5'-CTT 
AAAAAAAAGCCTTAACAGTACATGACAATCTCGAGA TTGTCATGTACTGTTAAGGC-3'. According to the manufacturer's instructions, the pPACKH1 HIV Lentivector Packaging kit (System Biosciences, LLC) was used to construct NCAPG-knockdown CRC cells. In short, $2.0 \mu \mathrm{g}$ shRNA-containing plasmids (corresponding control plasmid) and 5.0 $\mu \mathrm{g}$ pPACKH1 (3rd generation packaging system) packaging plasmid mix (pPACKH1-GAG, pPACKH1-REV and pVSV-G plasmids) were transfected into $70-80 \%$ confluent 293 T cells using Lipofectamine ${ }^{\circledR} 2000$ reagent (Invitrogen; Thermo Fisher Scientific, Inc,). After 24 h, PEG-it virus precipitation solution (System Biosciences) was added to the collected culture media, which was incubated overnight at $4^{\circ} \mathrm{C}$ to concentrate the viruses. The virion-containing culture medium was centrifuged at $72,000 \mathrm{x}$ g for $2 \mathrm{~h}$ at $4^{\circ} \mathrm{C}$. The virus particles were resuspended in $1 \mathrm{X}$ phosphate-buffered saline and aliquots were stored at $-80^{\circ} \mathrm{C}$. The viruses were transduced into HCT 15 or HCT 116 cells using polybrene $(8 \mu \mathrm{g} / \mathrm{ml}$; Sigma-Aldrich; Merck KGaA) at a multiplicity of infection of 20. Stably transfected HCT 15 or HCT 116 cells were selected via puromycin $(6 \mu \mathrm{g} / \mathrm{ml})$ in medium, and the maintenance at was $3 \mu \mathrm{g} / \mathrm{ml}$ puromycin. Successful transfection was confirmed using RT-qPCR and western blotting.

For miRNA inhibitor or mimic transfection, miR-23b-3p inhibitor (anti-miR-23b-3p, 5'-GGUAAUCCC UGGCAAUGUGAU-3'), miR-23b-3p mimic (sense 5'-AUC ACAUUGCCAGGGAUUACC-3'; and antisense, 5'-GGU AAUCCCUGGCAAUGUGAU-3') and their corresponding negative controls (anti-miR-negative control, 5'-GGUAAU CCCUGGCAAUGUGAU-3'; miR-negative control sense, 5'-UUUGUACUACACAAAAGUACUG-3' and antisense, 5'-CAGUACUUUUGUGUAGUACAAA-3') were obtained from Guangzhou RiboBio Co., Ltd. The open reading frame of NCAPG was amplified by PCR using primers that contain KpnI and EcoRI restrictions sites, and subcloned into vector pcDNA 3.1(+) (Invitrogen; Thermo Fisher Scientific, Inc.) to generate the construct pcDNA-NCAPG. Prior to transfection, $2 \times 10^{5}$ HCT 15 or HCT116 cells were seeded into 6-well plates and cultured at $37^{\circ} \mathrm{C}$ until reaching $60-70 \%$ confluence. The cells were subsequently transfected with $\sim 5 \mu$ g corresponding oligonucleotide (miR-296-3p or miR-NC mimics,) using Lipofectamine ${ }^{\circledR} 2000$ (Invitrogen; Thermo Fisher Scientific, Inc,). All cells were incubated at $37^{\circ} \mathrm{C}$ with $5 \% \mathrm{CO}_{2}$ for $48 \mathrm{~h}$ before subsequent experimentation.

Cellular proliferation assays. CRC cell proliferation was measured using colony formation and Cell Counting Kit-8 (CCK-8) assays. For the CCK-8 assay, following transfection, $\sim 3 \times 10^{3}$ cells/well were seeded into 96 -well culture plates and incubated at $37^{\circ} \mathrm{C}$ for $0,24,48,72$ or $96 \mathrm{~h}$. The cells were then incubated with CCK-8 reagent (Beyotime Institute of Biotechnology) for $1 \mathrm{~h}$. The optical density value of each well was measured using a spectrophotometer at a wavelength of $450 \mathrm{~nm}$. For the colony formation assay, $5 \times 10^{2}$ cells were seeded into 6 -well plates and cultured for $\sim 2$ weeks (until visible colonies had formed). Colonies were subsequently fixed with $4 \%$ paraformaldehyde and stained with $0.1 \%$ crystal violet, both for $15 \mathrm{~min}$ at room temperature. Stained colonies ( $>50$ cells) were counted using ImageJ software (version $1.53 \mathrm{c}$; National Institutes of Health).

Apoptosis analysis. Flow cytometry was performed to assess levels of apoptosis using an Annexin V-FITC Cell Apoptosis Detection kit (Beyotime Institute of Biotechnology). Briefly, transfected cells were harvested using trypsin-EDTA, washed twice with PBS and resuspended in binding buffer. Then, the prepared cells were stained with Annexin V-FITC and PI solutions according to the manufacturer's protocol. Apoptotic CRC cells were analyzed using a FACScan flow cytometer (BD Biosciences) and CellQuest software (version 6.0; BD Biosciences).

Xenograft tumor assay. A total of 12 female BALB/c nude mice (age, 1-2 months; Shanghai Laboratory Animal Center) were randomized into two groups (6 mice/group) and subcutaneously injected with HCT 15 or HCT 116 transfected with shNCAPG or sh control (Ctrl) CRC cell suspension $\left(1 \times 10^{6}\right.$ cells $\left./ \mathrm{ml}\right)$ in the right flank. All mice were sacrificed 4 weeks after the injection via $\mathrm{CO}_{2}$ inhalation (air displacement rate, $10-30 \%$ per minute), and the tumors were resected and weighed; the maximum tumor diameter was $<2 \mathrm{~cm}$. All mice were maintained under specific pathogen-free conditions with free access to water and sterile food, at $22-25^{\circ} \mathrm{C}$ and with a 12:12 h light/dark cycle, and the litters were changed every 2 days. The animal experimental protocol was approved by The Experimental Animal Ethics Committee of North Sichuan Medical College (approval no. 201927).

Hematoxylin-eosin $(H \& E)$ staining and immunohistochemical (IHC) staining. After resection from the nude mice, all tumors were fixed in $10 \%$ neutral formaldehyde solution at room temperature for $24 \mathrm{~h}$. Before staining, the tumors were dehydrated using a fully automatic tissue dehydrator (Leica Microsystems $\mathrm{GmbH}$ ) and embedded in paraffin using a tissue embedding system (Leica), prior to cutting into $3-\mu \mathrm{m}$ thick sections with an electronic paraffin slicer (Leica). The sections were then baked for $2 \mathrm{~h}$ at $60^{\circ} \mathrm{C}$ to prepare for staining. For H\&E staining: Briefly, after dewaxing and rehydration, the slices were stained with hematoxylin solution for $5 \mathrm{~min}$ at room temperature, then immersed in $1 \%$ acid ethanol $(1 \% \mathrm{HCl}$ in $70 \%$ ethanol) at room temperature for $5 \mathrm{~min}$ before rinsing in distilled water. The sections were then stained with eosin solution for $3 \mathrm{~min}$ and then dehydrated with graded alcohol and clarified in xylene, both at room temperature. The H\&E images were captured at $\mathrm{x} 400$ magnification using a digital light microscope camera system (Olympus Corporation).

For IHC staining, after dewaxing with xylene, the sections were rehydrated in a descending alcohol series $(100,95$ and $70 \%$, successively) and sequentially treated with $3 \%$ methanol hydrogen peroxide at room temperature for $10 \mathrm{~min}$. After washing three times with PBS, the slices were then treated with $10 \%$ citrate buffer $(\mathrm{pH}, 6.0)$ solution and blocked with $10 \%$ BSA for 20 min at room temperature. The prepared slices were incubated with Ki-67 primary antibody (1:100; cat. no. ab92742; Abcam) overnight at $4^{\circ} \mathrm{C}$. The next day, after being washed with PBS, the sections were incubated with an HRP-conjugated secondary antibody $(1: 5,000$; cat. 
no. ab7090; Abcam) for $1 \mathrm{~h}$ at $37^{\circ} \mathrm{C}$. Then, DAB solution (Beyotime Institute of Biotechnology) was used for staining for 5 min, accompanied by hematoxylin for counterstaining. Immunohistochemical images were captured using a digital light microscope camera system (Olympus Corporation). The Ki-67 expression scores of xenograft tumors were determined based on the ratio of positively-stained cells $(0,0-5 ; 1,6-35 ; 2,36-70$; and $3,>70 \%)$, and the staining intensity ( 0 , no staining; 1 , weakly stained; 2 , moderately stained; and 3 , strongly stained) was determined as previously described (17). The final score was determined by multiplying the percentage-positive score by the staining intensity score, and the final scores were defined as follows:,$- 0-1 ;+, 2-3 ;++, 4$ -6 ; and,$+++>6$. Low expression was defined as a total score $<4$, whereas high expression was defined as a total score $\geq 4$. The tissues were scored by two experienced pathologists.

Dual luciferase reporter assay. A Dual Luciferase Reporter assay system (Promega Corporation) was used to perform the dual luciferase reporter assay. Briefly, wild-type (WT) or mutant (MUT) 3'-UTR sequences of NCAPG were subcloned into the XhoI and NotI sites of the psiCHECK-2 vector (Promega Corporation), and the vectors were respectively named psiCHECK-2-NCAPG-WT and psiCHECK-2-NCAPG-MUT. The following primers were used to amplify the specific fragments: NCAPG-WT forward, 5'-CAACTCGAGGTGAGGAAGACCAGTATAACG-3' and reverse, 5'-CACAACACA AGCGGCCGCGTGTTTCGT TGGGTTTGCA-3'; and NCAPG-MUT forward, 5'-CAC AACTCGAGTGTGGATAAATTATCTAGACCGAATATT TACACTCAGGAACTTCTC-3' and reverse, 5'-CACAAC ACAAGCGGCCGCAAGTTCCTGAGTGTAAATATTCGG TTAGATAATTTATCCACAGTAA-3'. Then, 293T cells were seeded into 24 -well plates at a density of $2 \times 10^{4}$ cells/well, and transfected with $200 \mathrm{ng}$ psiCHECK-2-NCAPG-WT or psiCHECK-2-NCAPG-MUT vectors and $40 \mathrm{nM}$ anti-miR-23b-3p using Lipofectamine ${ }^{\circledR} 2000$. Following $48 \mathrm{~h}$ of transfection, the cells were harvested, and the relative luciferase activity was measured using the Dual Luciferase Reporter assay system, according to the manufacturer's protocol. Firefly luciferase activity was normalized to that of Renilla luciferase.

Bioinformatics analysis. To verify the expression of NCAPG and miR-23b-2p in CRC and normal tissues, Oncomine (https://www.oncomine.org), Gene Expression Profiling Interactive Analysis (GEPIA; http://gepia.ancer-pku.cn/index. html) and the database of Differentially Expressed miRNAs in human Cancers (dbDEMC; version 2.0; https://www.picb. ac.cn/dbDEMC/index.html) were used. In addition, the Human Protein Atlas (HPA; https://www.proteinatlas.org) was used to obtain immunohistochemistry staining images of NCAPG expression in CRC tissues. To validate the survival analysis results of high and low NCAPG expression in patients with CRC, a survival curve was generated using GenomicsScape (http://www.genomicscape.com). The correlation between NCAPG and PI3K or AKT gene family member expression was downloaded from the GEPIA database, and the binding relationship between NCAPG and miR-23b-3p was identified using StarBase (http://starbase.sysu.edu.cn). Moreover,
Table I. Relationship between NCAPG and the clinicopathological features of 83 patients with colorectal cancer.

\begin{tabular}{|c|c|c|c|}
\hline \multirow[b]{2}{*}{ Variable } & \multicolumn{2}{|c|}{ NCAPG expression } & \multirow[b]{2}{*}{ P-value } \\
\hline & $\begin{array}{c}\text { Low } \\
(n=41)\end{array}$ & $\begin{array}{c}\text { High } \\
(n=42)\end{array}$ & \\
\hline Age & & & 0.340 \\
\hline$<60$ & 14 & 10 & \\
\hline$\geq 60$ & 27 & 32 & \\
\hline Sex & & & 0.827 \\
\hline Male & 21 & 20 & \\
\hline Female & 20 & 22 & \\
\hline T stage & & & 0.826 \\
\hline $\mathrm{T} 1$ and $\mathrm{T} 2$ & 23 & 25 & \\
\hline $\mathrm{T} 3$ and $\mathrm{T} 4$ & 18 & 17 & \\
\hline Lymph node metastasis & & & 0.383 \\
\hline Absent & 24 & 22 & \\
\hline Present & 17 & 20 & \\
\hline Differentiation & & & $0.006^{\mathrm{a}}$ \\
\hline Well & 18 & 10 & \\
\hline Moderate & 16 & 11 & \\
\hline Poor & 7 & 21 & \\
\hline Tumor size & & & $0.026^{\mathrm{a}}$ \\
\hline$<5 \mathrm{~cm}$ & 29 & 19 & \\
\hline$\geq 5 \mathrm{~cm}$ & 12 & 23 & \\
\hline Tumor location & & & 0.625 \\
\hline Colon & 12 & 10 & \\
\hline Rectum & 29 & 32 & \\
\hline
\end{tabular}

${ }^{\mathrm{a}} \mathrm{P}<0.05$. NACPG, non-structural maintenance of chromosomes condensin I complex subunit G.

TargetScan (http://www.targetscan.org), StarBase and miRcode (http://www.mircode.org/index.php) were used to predict the targeting relationship between miR-23b-3p and NCAPG.

Statistical analysis. Statistical analysis was performed using SPSS 21.0 software (IBM Corp). All experiments were performed at least three times, and continuous data are presented as the mean \pm SD. Statistical differences between two groups were determined using unpaired Student's t-test, though differences between paired tumor and adjacent tissues were determined by paired t-test. Differences between multiple groups were assessed by one-way ANOVA with Dunnett's or Tukey's post hoc test, and the $\chi^{2}$ test was employed to analyze categorical data. The postoperative survival rate of patients with CRC was analyzed using the Kaplan-Meier method, and the differences in survival rates between groups were statistically analyzed using the log-rank test. Univariate and multivariate Cox proportional hazard model analyses were applied to identify independent prognostic factors of NCAPG in overall survival (OS) rate. The correlation between NCAPG 
Table II. Univariate and multivariate Cox regression analysis of the clinicopathological factors for overall survival from clinical information of patients with CRC.

\begin{tabular}{|c|c|c|c|c|c|c|}
\hline \multirow[b]{2}{*}{ Variable } & \multicolumn{2}{|c|}{ Univariate analysis } & \multirow[b]{2}{*}{ P-value } & \multicolumn{2}{|c|}{ Multivariate analysis } & \multirow[b]{2}{*}{ P-value } \\
\hline & HR & $95 \% \mathrm{CI}$ & & HR & $95 \% \mathrm{CI}$ & \\
\hline Age & 2.326 & $0.798-6.780$ & 0.122 & & & \\
\hline Sex & 0.705 & $0.320-1.555$ & 0.387 & & & \\
\hline T stage & 2.295 & $1.030-5.111$ & $0.042^{\mathrm{a}}$ & 2.548 & $1.130-5.747$ & $0.024^{\mathrm{a}}$ \\
\hline Lymph node metastasis & 3.514 & $1.467-8.415$ & $0.005^{\mathrm{a}}$ & 3.225 & $1.143-9.100$ & $0.027^{\mathrm{a}}$ \\
\hline Differentiation & 1.758 & $1.054-2.930$ & $0.031^{\mathrm{a}}$ & 1.068 & $0.575-1.984$ & 0.834 \\
\hline Tumor size & 2.217 & $0.995-4.939$ & 0.051 & & & \\
\hline Tumor location & 1.340 & $0.502-3.576$ & 0.558 & & & \\
\hline NCAPG expression & 3.357 & $1.340-8.412$ & $0.010^{\mathrm{a}}$ & 3.095 & $1.095-8.747$ & $0.033^{\mathrm{a}}$ \\
\hline
\end{tabular}

${ }^{\text {aP }}<0.05$. HR, hazard ratio; CI, confidence interval; NACPG: Non-structural maintenance of chromosomes condensin I complex subunit G.

and miR-23b-3p expression was determined using Spearman's rank correlation analysis. $\mathrm{P}<0.05$ was considered to indicate a statistically significant difference.

\section{Results}

NCAPG expression in CRC tissues and cell lines. The Oncomine and GEPIA databases were used to determine NCAPG expression levels in CRC and adjacent normal tissues. The results revealed that NCAPG expression levels were significantly upregulated in CRC tissues (Fig. 1A and B). In addition, the HPA database indicated that NCAPG expression was high in $\mathrm{CRC}$ tissues, as determined using immunohistochemistry (Fig. 1C). Furthermore, NCAPG expression levels were found to be upregulated in the collected primary CRC tissues compared with those in the corresponding normal tissues (Fig. 1D). Similar outcomes were observed in the CRC cell lines (Fig. 1E). Therefore, the results suggested that the expression levels of NCAPG was upregulated in CRC tissues and cell lines.

Association between NCAPG expression and the clinicopathological characteristics of patients with CRC. To determine the association between NCAPG expression and patient clinicopathological features, patients with $\mathrm{CRC}$ were divided into high $(n=42)$ and low $(n=41)$ expression groups based on the median NCAPG expression value. As illustrated in Table I, high expression of NCAPG was closely associated with poor differentiation and a large tumor size. A survival curve was plotted according to the survival status and follow-up time, and showed that high NCAPG expression was associated with a shorter OS time (Fig. 1F). This result was also verified via Kaplan-Meier survival analysis using data from the GenomicsScape database (Fig. 1G). Furthermore, Cox proportional hazard regression analyses were performed to identify independent prognostic markers of OS. Since T stage and lymph node metastasis determine TNM stage in CRC (18), TNM stage was not included in Cox analysis in the present study. The results revealed that $\mathrm{T}$ stage, lymph node metastasis and NCAPG expression were independent prognostic factors (Table II), indicating that NCAPG may be involved in the progression of CRC, and may be useful for predicting the OS of patients.

Effect of NCAPG on CRC cell proliferation in vitro and in vivo. Next, the effects of NCAPG expression on CRC cell proliferation were investigated. The knockdown efficiency of NCAPG was confirmed using western blotting (Figs. 2A and S1A) and RT-qPCR (Fig. 2B). As shown in Fig. 2C and D, NCAPG-knockdown markedly reduced the colony formation and proliferative capacity of HCT15 and HCT116 CRC cells. Subsequently, to further determine the effect of NCAPG on tumor growth, CRC xenograft models were constructed using $\mathrm{BALB} / \mathrm{c}$ nude mice. The results revealed that the mean weight of xenograft tumors in the shNCAPG group was notably reduced compared with that in the shCtrl group (Fig. 2E and F). To further verify the characteristics of nude mice xenograft tumors, H\&E and IHC staining were performed. The results of $\mathrm{H} \& \mathrm{E}$ staining demonstrated that the tumor cells proliferated vigorously and were arranged irregularly (Fig. S2A). In addition, the IHC staining results showed the ki-67 expression in xenograft tumors, and that the expression in the shCtrl group was higher than in the shNCAPG group (Fig. S2B), confirming that the xenograft tumors are derived from CRC cells. These results suggested that NCAPG-knockdown inhibited CRC cell proliferation both in vitro and in vivo.

Effect of NCAPG on CRC cell apoptosis. To investigate the role of NCAPG on CRC cell apoptosis, the expression levels of apoptosis-related proteins, Bcl-2 and Bax, were detected in CRC cells. The results demonstrated that Bcl-2 expression was downregulated, while Bax expression was upregulated following NCAPG-knockdown in CRC cell lines (Figs. 2G and S1B). The results of flow cytometry analysis revealed that silencing NCAPG increased the percentage of cells undergoing apoptosis compared with the shCtrl group (Fig. 2H). These data suggest that NCAPG suppressed the apoptosis of CRC cells.

NCAPG regulates the PI3K/AKT signaling pathway in CRC cells. The PI3K/AKT signaling pathway has been found to 
A

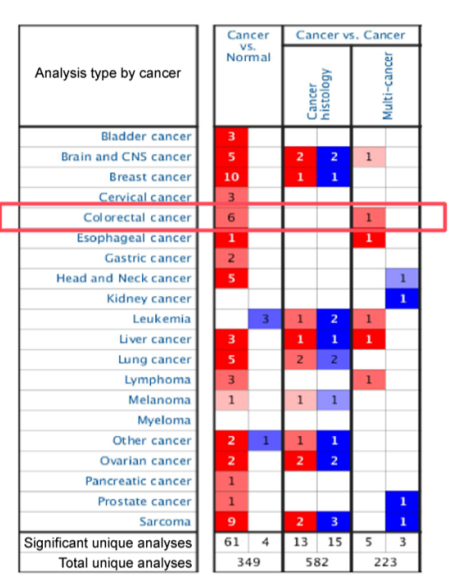

D

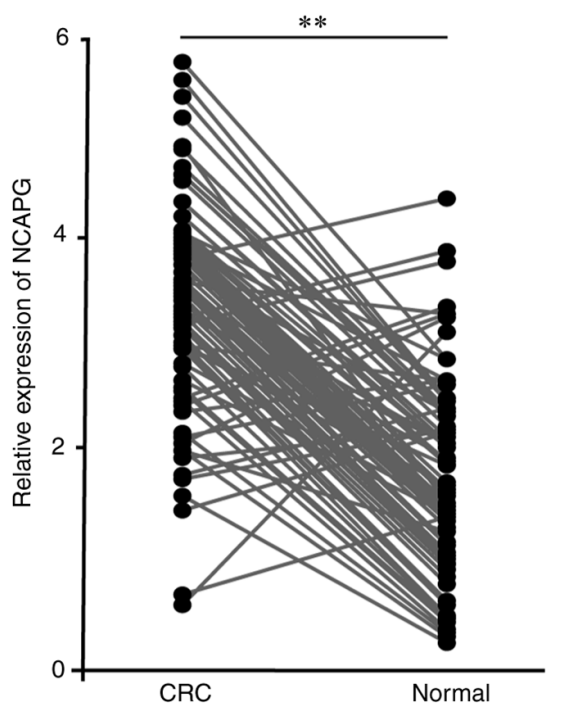

$\mathrm{F}$

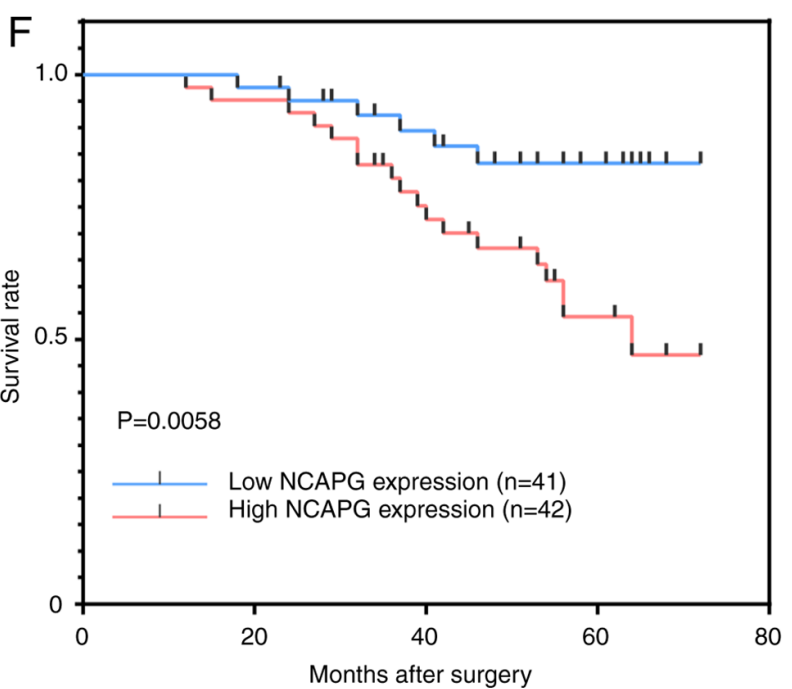

C

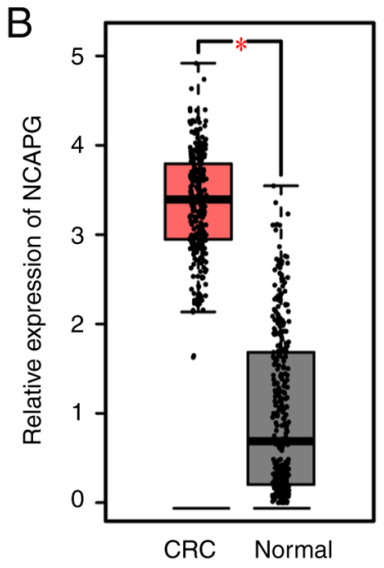

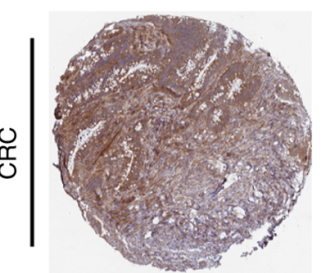
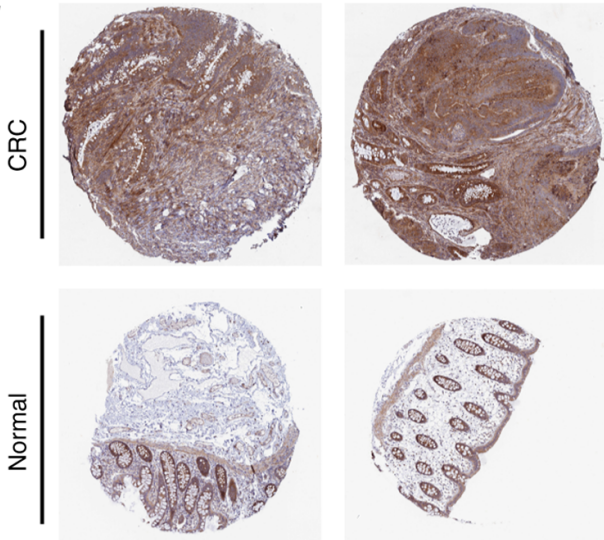

E

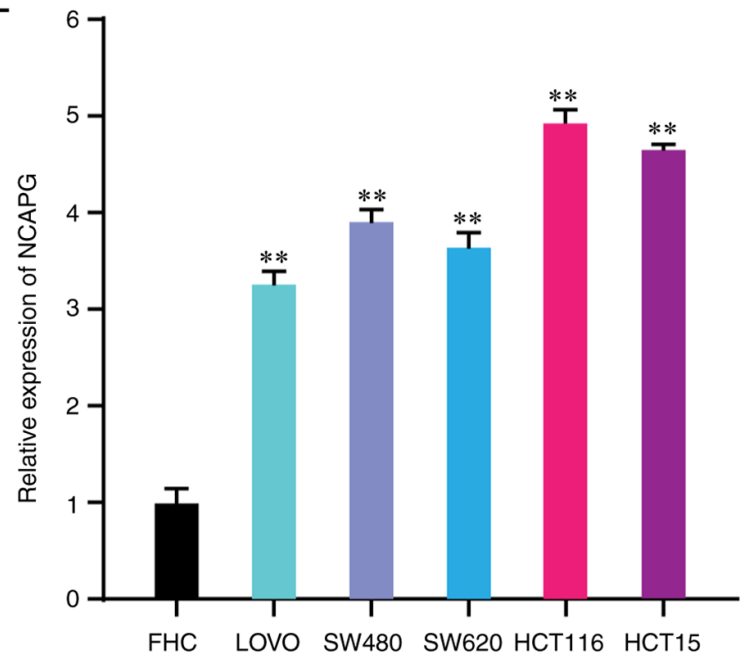

G

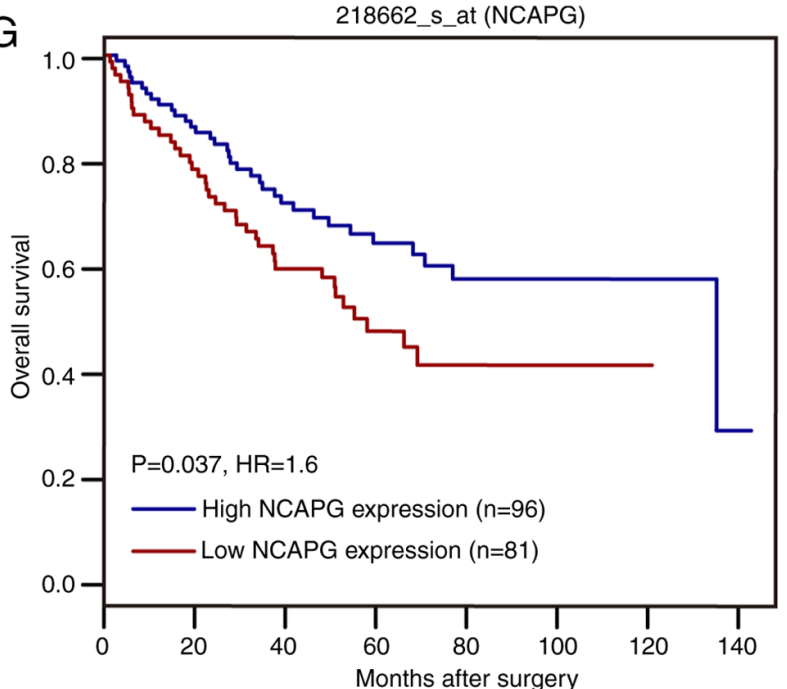

Figure 1. NCAPG expression levels are upregulated in CRC tissues and cell lines, and are associated with poor differentiation, large tumor size and shorter overall survival times in CRC. (A) NCAPG expression in all cancer cases obtained from the Oncomine database. (B) NCAPG expression in CRC and normal tissues downloaded from the Gene Expression Profiling Interactive Analysis database. (C) Results of the immunohistochemistry analysis of NCAPG expression in normal colorectal and CRC tissues acquired from the Human Protein Atlas. (D) Relative expression of NCAPG in CRC tumor and adjacent normal tissues. (E) Relative expression of NCAPG in the normal colon cell line, FHC and CRC cells. (F) Kaplan-Meier survival curve based on NCAPG expression status in patients with CRC. (G) Kaplan-Meier survival curve of patients with CRC based on NCAPG expression data from the GenomicsScape database. ${ }^{*} \mathrm{P}<0.05$ and $^{* *} \mathrm{P}<0.01$. NCAPG, non-SMC condensing I complex subunit G; CRC, colorectal cancer.

exert crucial effects on the biological progression of various types of cancer (19). Previous research has revealed that the
PI3K/AKT signaling pathway is modulated by NCAPG in gastric cardia adenocarcinoma (7) and HCC (9). Thus, we 
A

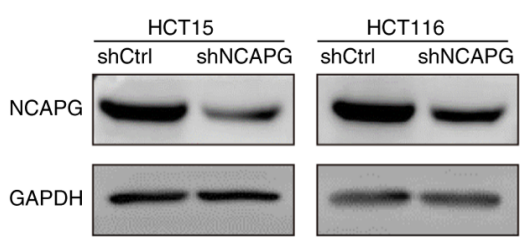

B

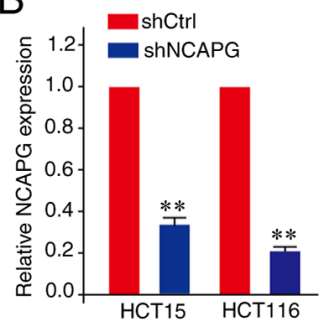

C

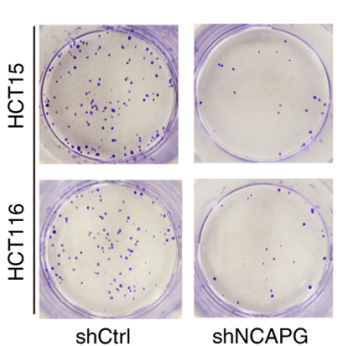

E

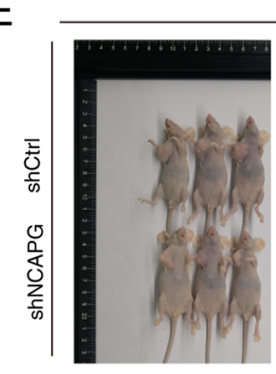

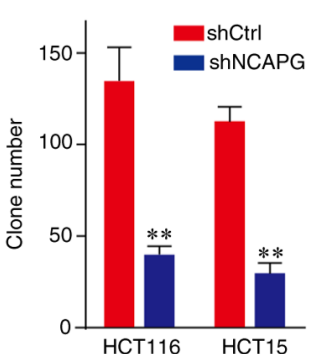

HCT15
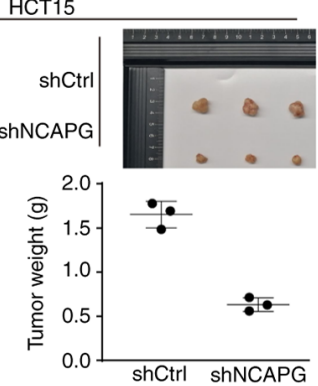

F

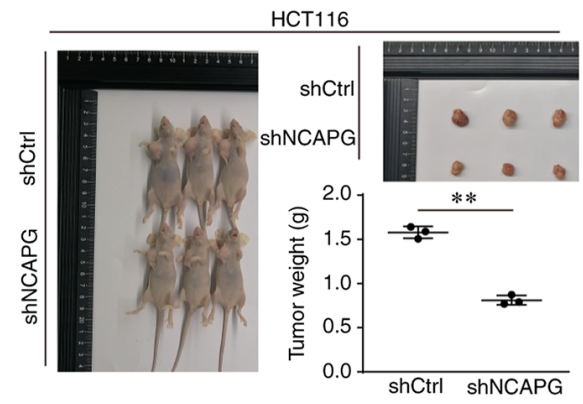

G
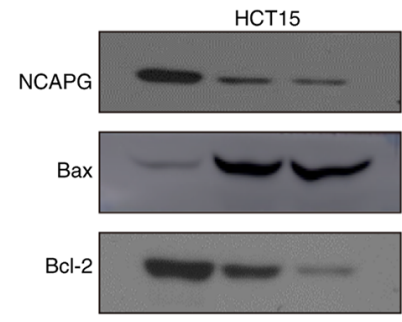

GAPDH

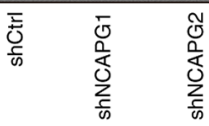
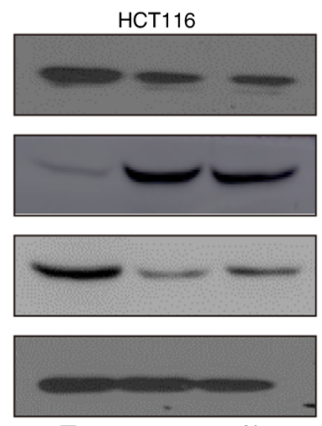

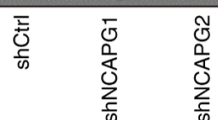
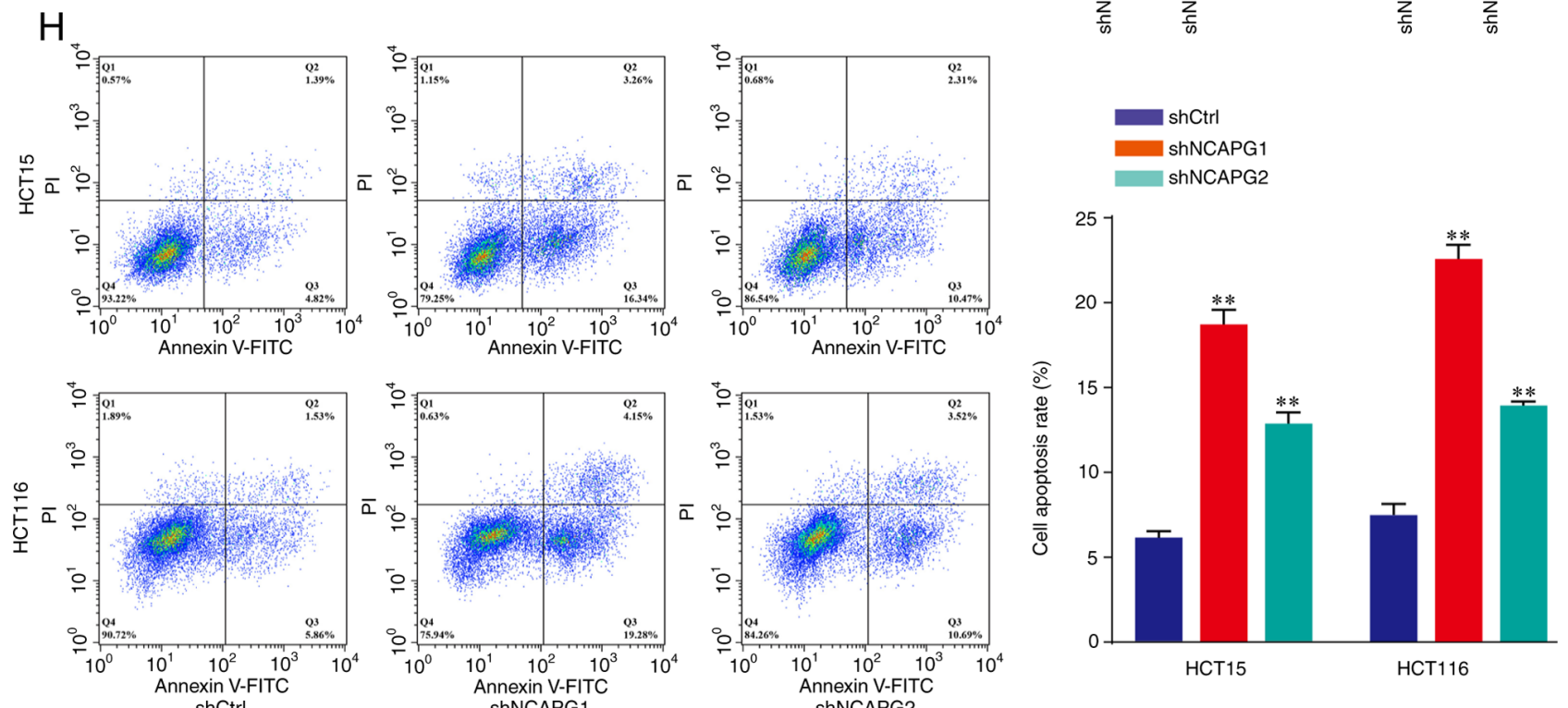

Figure 2. Effect of NCAPG on CRC cell proliferation and apoptosis. (A) Western blotting and (B) reverse transcription-quantitative PCR were used to detect the expression levels of NCAPG in CRC cells transfected with shCtrl or shNCAPG. (C) Colony formation and (D) Cell Counting Kit-8 assays were performed to determine the proliferative ability of untransfected and transfected CRC cells. (E and F) Representative images of the formation of xenograft tumors in BALB/c nude mice and the corresponding excised tumors in the shCtrl and shNCAPG groups. (G) Western blotting was used to determine the effect of NCAPG-knockdown on the expression levels of the apoptosis-related proteins, Bcl-2 and Bax. (H) Effect of NCAPG-knockdown on CRC cell apoptosis was assessed using flow cytometry. ${ }^{* *} \mathrm{P}<0.01$. NCAPG, non-SMC condensing I complex subunit G; CRC, colorectal cancer; sh, short hairpin RNA; Ctrl, control.

hypothesized that NCAPG may regulate the PI3K/AKT signaling pathway in CRC. The GEPIA database revealed that NCAPG expression was positively correlated with the expression of the PI3K/AKT gene family members, 
A
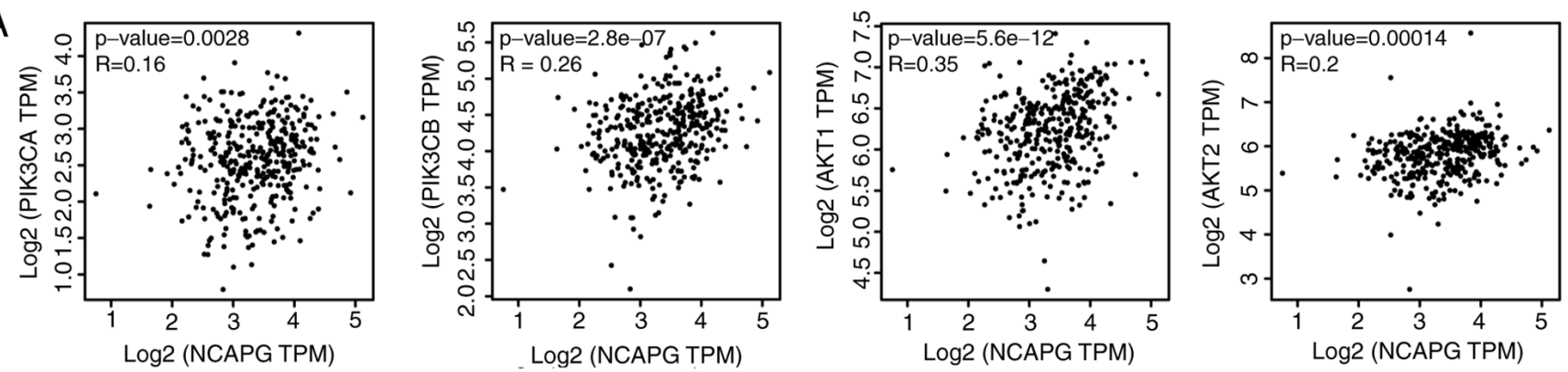

B

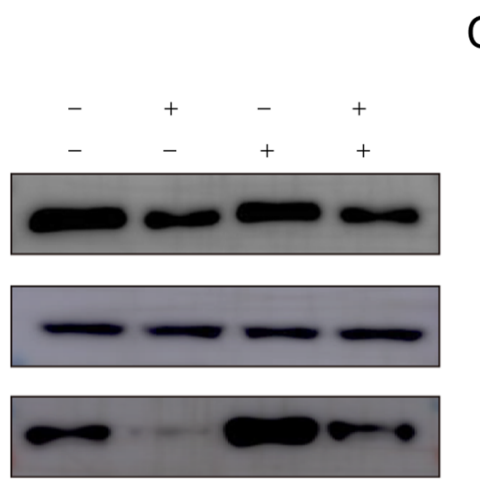

$\mathrm{C}$
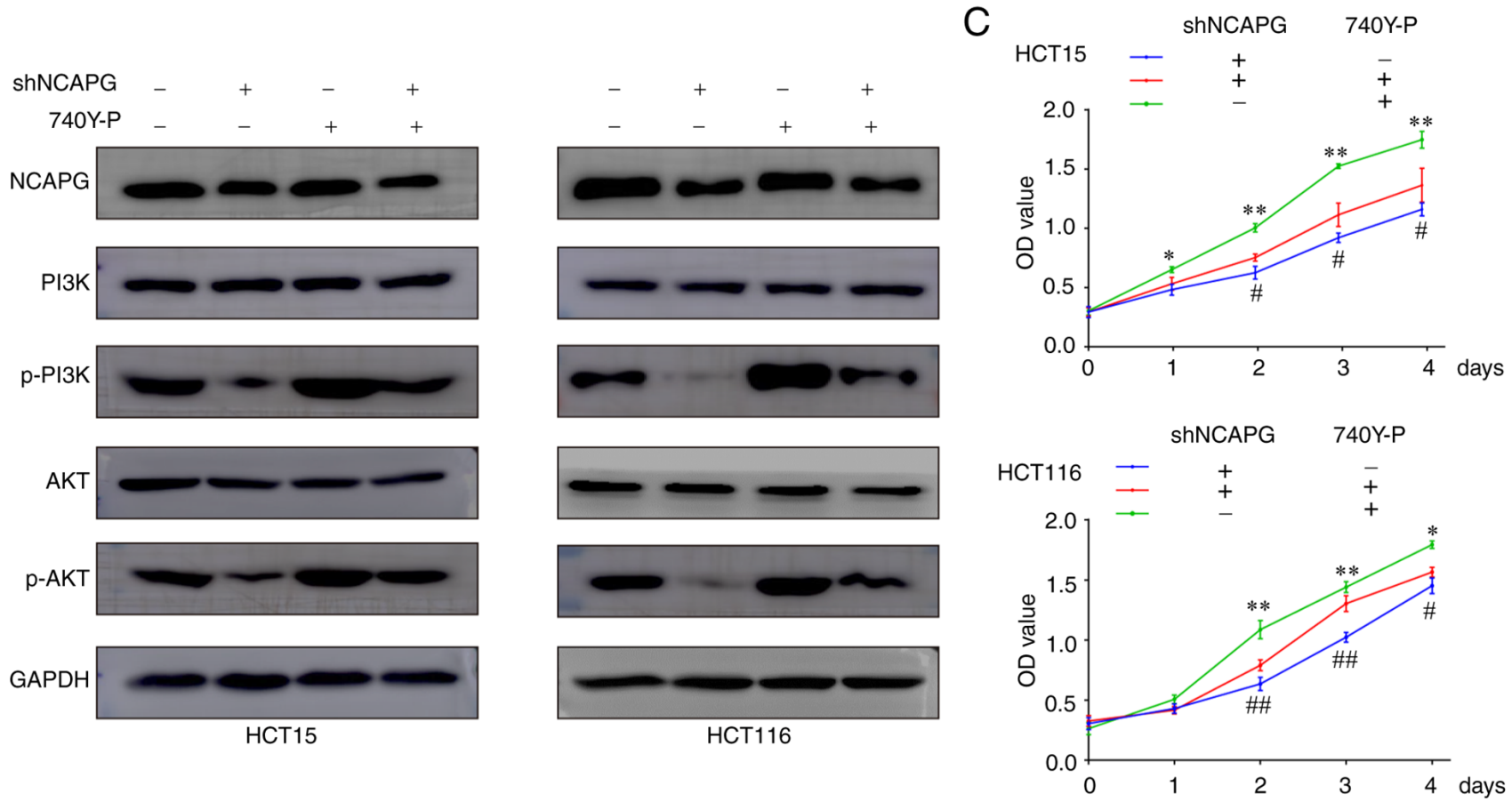

$\mathrm{D}$
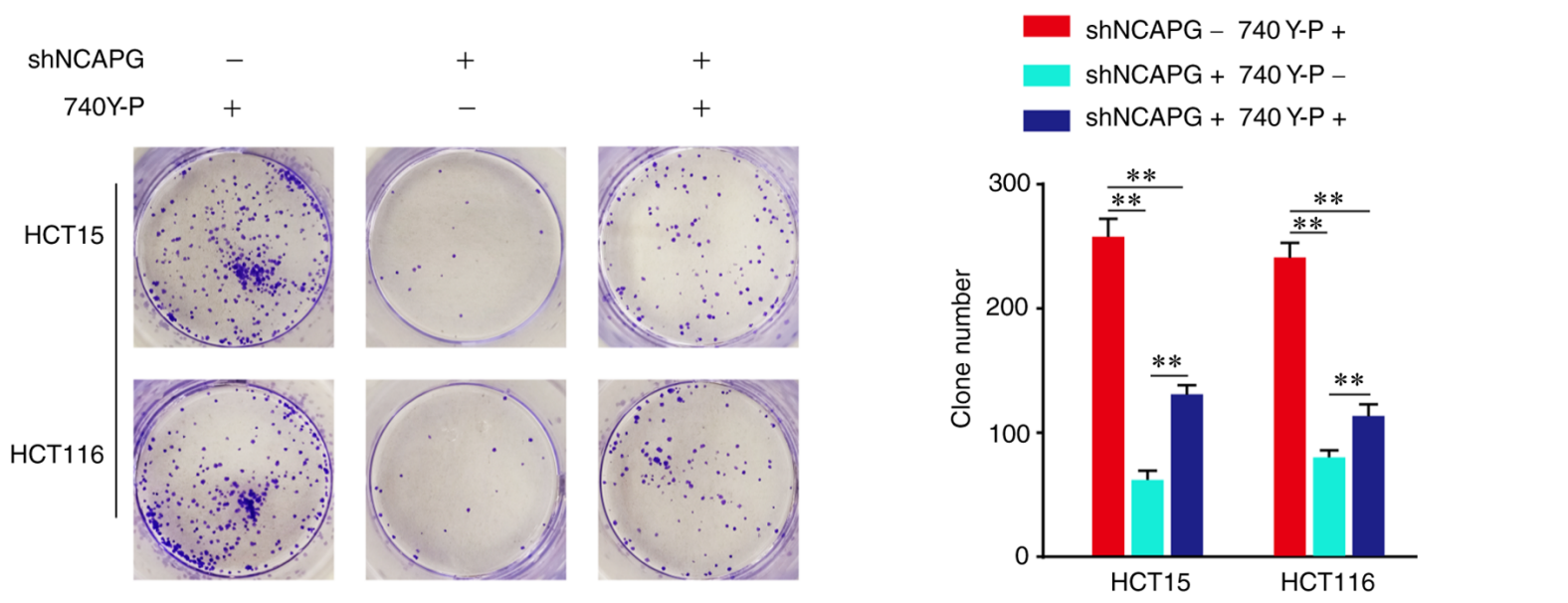

Figure 3. NCAPG regulates the PI3K/AKT signaling pathway in CRC cells. (A) Correlation between NCAPG and PI3K/AKT gene family members at the mRNA expression level was determined using the Gene Expression Profiling Interactive Analysis database. (B) Western blotting was used to analyze the relative protein levels of PI3K, p-PI3K, AKT and p-AKT in transfected and 740Y-P-treated CRC cells. (C) Cell Counting Kit- 8 and (D) colony formation assays were performed to validate the proliferative ability of transfected $\mathrm{CRC}$ cells. ${ }^{*} \mathrm{P}<0.05$ and ${ }^{* *} \mathrm{P}<0.01 \mathrm{vs}$. shNCAPG $+/ 740 \mathrm{Y}-\mathrm{P}+;{ }^{*} \mathrm{P}<0.05$ and ${ }^{\# \#} \mathrm{P}<0.01$ vs. shNCAPG +/740Y-P. NCAPG, non-SMC condensing I complex subunit G; CRC, colorectal cancer; $\mathrm{p}-$, phosphorylated.

phosphatidylinositol-3,5-bisphsphate 3-kinase catalytic subunit, phosphatidylinositol-4,5-bisphosphate 3-kinase catalytic subunit, AKT1 and AKT2 (Fig. 3A). Moreover, western blotting demonstrated that both p-PI3K and p-AKT levels were significantly downregulated following NCAPG-knockdown in CRC cells, while treatment with 740Y-P (an activator of PI3K) reversed the suppressive effects of shNCAPG on p-PI3K and p-AKT expression (Figs. 3B and S1C). In addition, treatment with 740Y-P rescued the effects of shNCAPG on cellular proliferation (Fig. $3 \mathrm{C}$ and D). These findings suggest that NCAPG regulated CRC cell proliferation via the PI3K/AKT signaling pathway. 
A

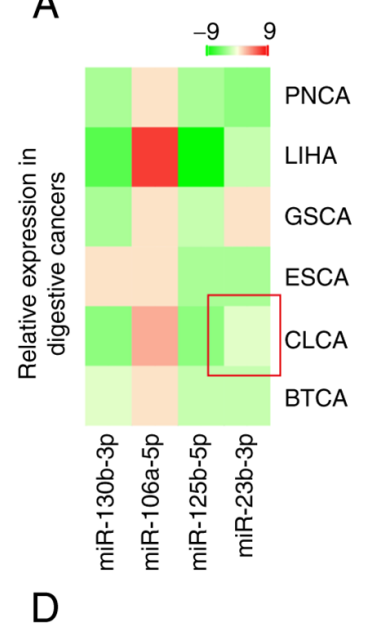

B hsa-miR-23b-3p vs. NCAPG, 450 samples (COAD)

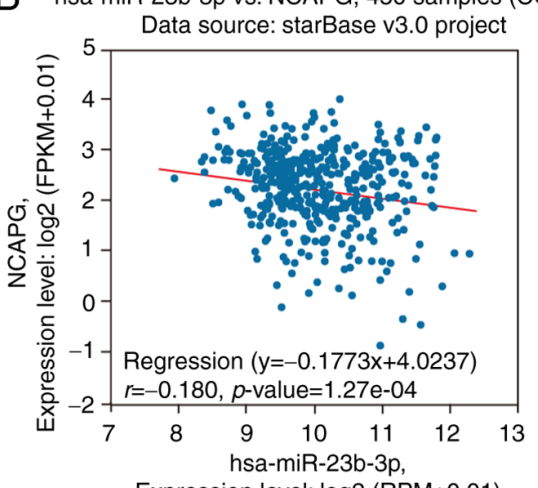

C

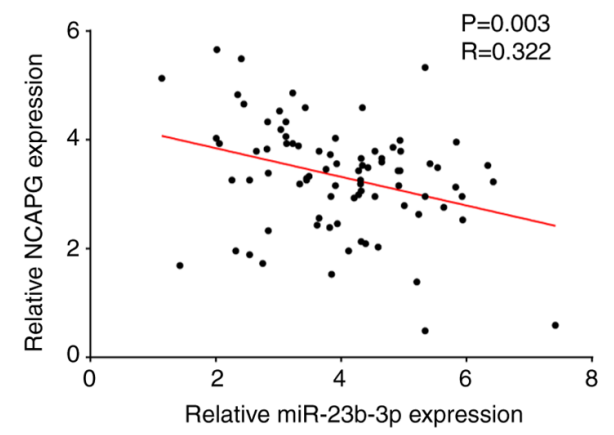

Hsa-miR-23b-3p 3' CCAUUAGGG- -ACC- - - -GUUACACUA 5'

\|\| $\mid \|$ aAd $\||\|\mid\|$

NCAPG WT 5' AAUAAUUCUAGUGGGAAUAAAUGUGAC 3’

NCAPG Mut 5' AAAUUAUCUAGACCGAAUAUUACACUC 3'
E

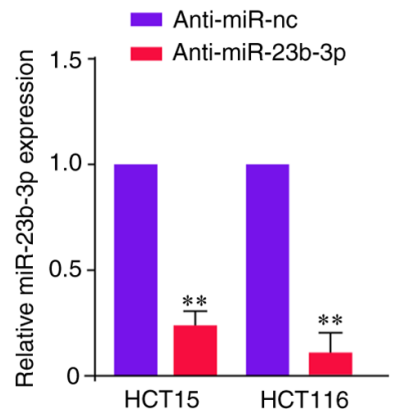

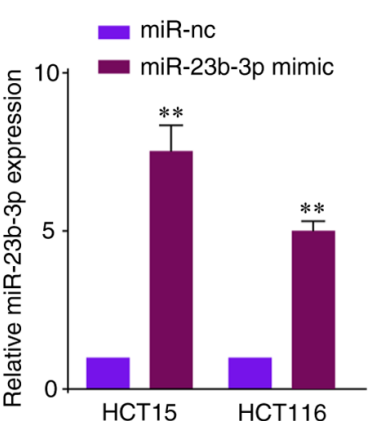

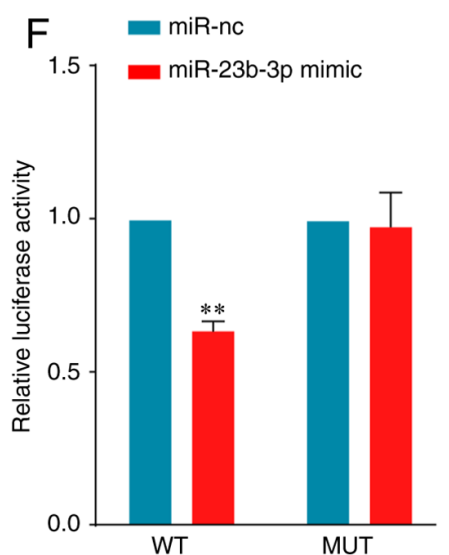

$\mathrm{H}$

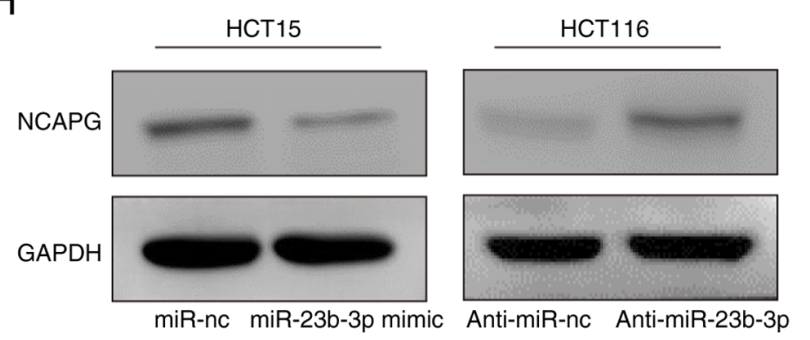

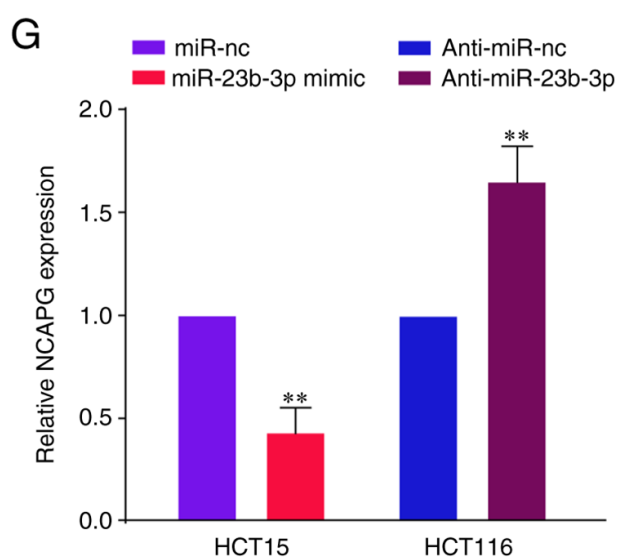
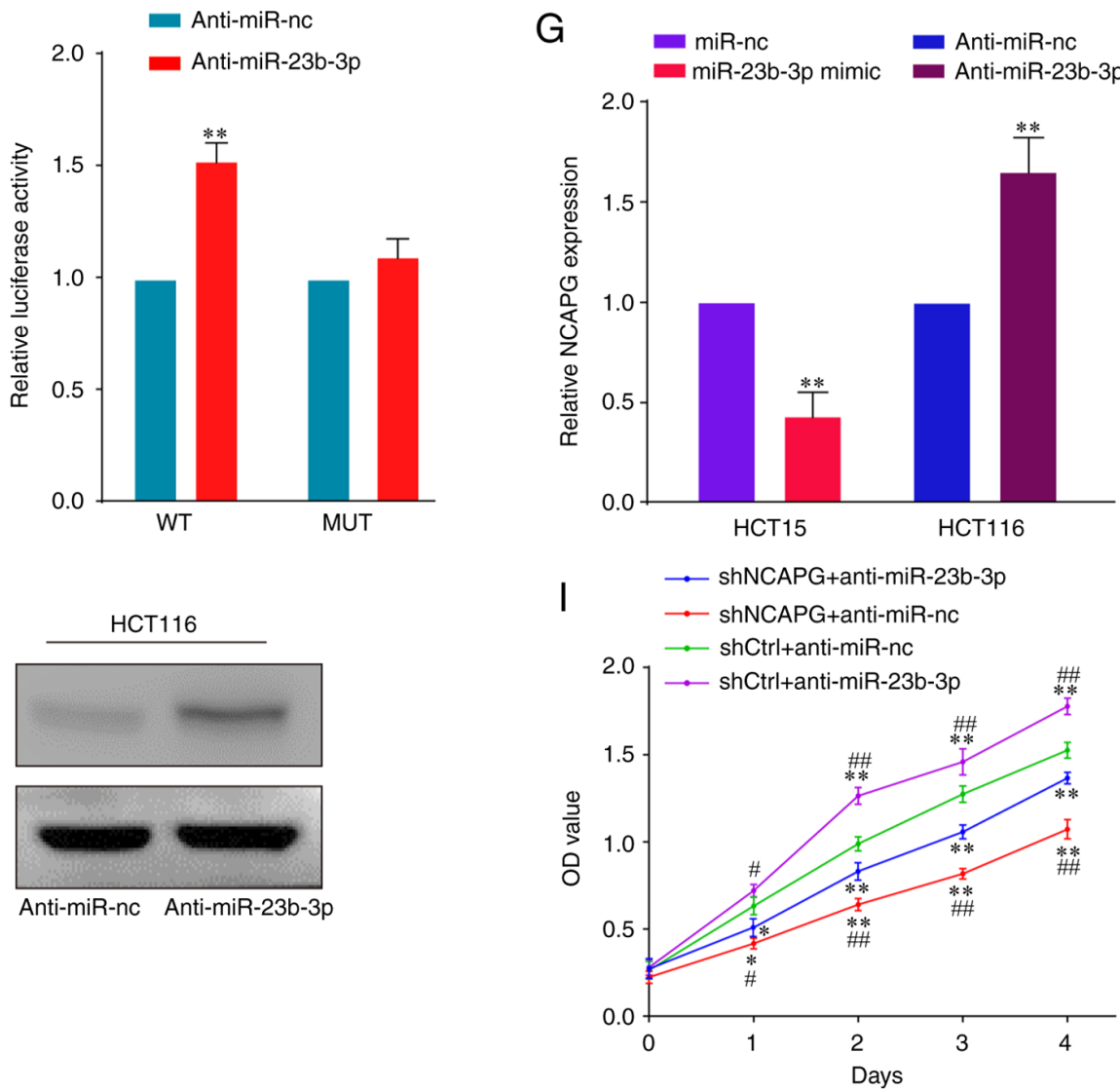

Figure 4. miR-23b-3p directly targets NCAPG in CRC cells. (A) Heatmap of miR-23b-3p expression in cancers of the digestive system was obtained from the database of Differentially Expressed miRNAs in human Cancers 2.0. (B) Correlation between miR-23b-3p and NCAPG expression in 450 patients with CRC based on data from the StarBase database. (C) Association between NCAPG and miR-23b-3p expression in the present study patient cohort. (D) Predicted binding sites between miR-23b-3p and NCAPG and WT and MUT putative miR-23b-3p target sequences in the 3'-UTR of NCAPG. (E) RT-qPCR was used to determine the relative expression levels of miR-23b-3p in CRC cells transfected with anti-miR-23b-3p or miR-23b-3p mimic. (F) Relative luciferase activity of psiCHECK-2-NCAPG-WT and psiCHECK-2-NCAPG-MUT vectors co-transfected into HCT116 cells with the miR-23b-3p mimic or anti-miR-23b-3p. (G) RT-qPCR and (H) western blotting were used to detect NCAPG expression levels in HCT15 or HCT116 cells. (I) Cell Counting Kit-8 assay was used to determine the proliferative ability of HCT116 cells in the rescue experiments. ${ }^{~} \mathrm{P}<0.05$ and ${ }^{* *} \mathrm{P}<0.01$ vs. shCtrl + anti-miR-nc. ${ }^{~} \mathrm{P}<0.05$ and ${ }^{\# \#} \mathrm{P}<0.01$ vs. shNCAPG + anti-miR-23b-3p. microRNA; NCAPG, non-SMC condensing I complex subunit G; CRC, colorectal cancer; WT, wild-type; MUT, mutant; UTR, untranslated region; RT-qPCR, reverse transcription-quantitative PCR; anti-miR-23b-3p, miR-23b-3p inhibitor. 
miR-23b-3p directly targets NCAPG in CRC cells. Previous studies on HCC (20) and prostate cancer (8) have demonstrated that NCAPG expression can be regulated by miRNAs. The publicly available databases, StarBase, TargetScan and miRcode, were used to predict that NCAPG was a possible target of miR-23b-3p (Fig. S3). Moreover, the heatmap downloaded from the dbDEMC suggested that miR-23b-3p expression was downregulated in CRC (Fig. 4A). Notably, a negative relationship between the expression levels of NCPAG and miR-23b-3p was identified using StarBase (Fig. 4B), which was consistent with the findings obtained using the present study patient cohort (Fig. 4C).

To validate the direct binding of miR-23b-3p to its target NCAPG in CRC, dual luciferase reporter assays were performed. First, the binding sites between miR-23b-3p and NCAPG mRNA were predicted using bioinformatics analysis (Fig. 4D). Then, RT-qPCR was used to verify the knockdown and overexpression efficiencies of miR-23b-3p (Fig. 4E). The results of the dual luciferase reporter assay in HCT116 cells revealed that miR-23b-3p overexpression significantly decreased the relative luciferase activity, whereas miR-23b-3p inhibition increased the relative luciferase activity (Fig. 4F). In addition, a mutation in the miR-23b-3p binding site in the NCAPG 3'-UTR abrogated both the function of the miR-23b-3p mimics and anti-miR-23b-3p, suggesting that miR-23b-3p may bind to the 3'-UTR of NCAPG. Moreover, RT-qPCR (Fig. 4G) and western blot (Figs. 4H and S1D) analyses indicated that NCAPG expression levels were significantly downregulated in CRC cells overexpressing miR-23b-3p compared with those in cells transfected with anti-miR-23b-3p. To determine whether miR-23b-3p exerted its function by regulating NCAPG, rescue experiments were performed using HCT116 cells. As shown in Fig. 4I, HCT116 cells transfected with anti-miR-23b-3p exhibited increased levels of proliferation compared with transfected anti-miR-NC CRC cells, suggesting that the co-transfection of anti-miR-23b-3p may partially reverse the inhibitory effects induced by shNCAPG in CRC cells. These results suggest that miR-23b-3p affected CRC cell proliferation by directly targeting NCAPG.

\section{Discussion}

Accumulating evidence has revealed that the upregulated expression of NCAPG is associated with several types of malignant tumor, including gastric cancer (6-7), HCC (9) and ovarian cancer (10). The results of the current study demonstrated that the expression levels of NCAPG were upregulated in CRC tissues and cell lines, which is consistent with the findings of our previous study (11). In another previous study, upregulated NCAPG expression was found to be associated with TNM stage, distant metastasis, nodal status and vascular invasion in gastric cancer (6). The current study results demonstrated that upregulated NCAPG expression levels were associated with poor differentiation, large tumor size and a shorter OS in patients with CRC, which indicated that NCAPG may be involved in the occurrence and development of CRC, and may represent a potential prognostic biomarker therein. Previous studies have also reported that NCAPG serves as an oncogene to promote tumor growth and metastasis in various types of cancer (5-7,9). Similarly, the results of the present study revealed that NCAPG significantly promoted CRC cell tumorigenesis and inhibited apoptosis in vitro and in vivo. Thus, NCAPG may exert a pro-carcinogenic effect in CRC.

To investigate the molecular mechanisms underlying the effects of NCAPG in CRC, the PI3K/AKT signaling pathway was selected and investigated in more detail, as it has been reported to be regulated by NCAPG in several cancer types $(7,9)$. The PI3K/AKT signaling pathway performs crucial roles in a number of cellular processes (21), and is reportedly involved in the genesis and progression of various types of tumor (22). The carcinogenesis of CRC occurs in a multi-step manner, which is mediated by the abnormal regulation of several pathways, including the PI3K/AKT signaling cascade (23). When the activated form of PI3K binds to the AKT pleckstrin homology domain, downstream signaling events not only mediate the translocation of AKT from the cytoplasm to the membrane, but also accelerate its conformational change (23). Activated AKT has been discovered to further affect the invasion, migration, proliferation and apoptosis of cancer cells (23). The results of the present study demonstrated that NCAPG-knockdown markedly downregulated the phosphorylation levels of PI3K and AKT. However, treatment with a PI3K signaling activator (740Y-P) rescued the effects induced by shNCAPG on the aforementioned cellular processes. Moreover, activation of the PI3K/AKT signaling pathway impaired the NCAPG-induced suppression of CRC cell proliferation. Thus, these findings suggest that the NCAPG-induced suppression of CRC cell proliferation may occur via inhibition of the PI3K/AKT signaling pathway.

miRNAs regulate gene expression at the post-transcriptional level, and an increasing number of research groups have begun to focus on the role of miRNAs in the carcinogenesis and development of cancers (24). NCAPG was found to be negatively regulated by miR-181c in HCC (19), and by miR-99a-3p in prostate cancer (8). However, to the best of our knowledge, there has been no evidence to suggest that miRNAs may regulate NCAPG expression in CRC cells. In the present study, miRNAs upstream of NCAPG were predicted via bioinformatics analysis, and miR-23b-3p was selected as the candidate miRNA. miR-23b-3p is known to be an important modulator of cellular physiology, and has been strongly associated with cancer development (14); In fact, the expression levels of miR-23b-3p were reported to be downregulated in cervical (25), lung (26) and prostate cancer (27). Consistent with the results of these aforementioned studies, the findings of the current study revealed that miR-23b-3p expression was downregulated in CRC tissues. However, other studies have reported that miR-23b-3p expression is upregulated in several types of malignant tumor, including renal (28), lung (29) and pancreatic cancer $(30,31)$. The discrepancy in these results may be due to differences in both the tissue types and the disease in question. Moreover, the results of the present study revealed that NCAPG and miR-23b-3p expression levels were inversely correlated. Dual luciferase reporter assays, western blotting and cellular proliferation assays further confirmed this targeting and modulatory relationship. These findings provide sufficient evidence to suggest that NCAPG may be directly targeted by miR-23b-3p in CRC cells. However, the present study has several limitations. Firstly, the precise mechanism underlying the regulatory effects of NCAPG in CRC remains 
unclear. Secondly, the present study was a single institute study and the number of samples used was small.

In conclusion, the findings of the current study suggested that NCAPG may promote the proliferation and inhibit the apoptosis of CRC cells by regulating the PI3K/AKT signaling pathway, and that these effects were modulated by miR-23b-3p. These results indicate that NCAPG may act as an oncogene and represent a potential prognostic marker in CRC.

\section{Acknowledgements}

Not applicable.

\section{Funding}

The present study was funded by the Scientific and Technological Cooperation Project of Nanchong City (grant no. 18SXHZ0577), the Key Scientific Project of The Affiliated Hospital of North Sichuan Medical College (grant no. 19ZD004), the Key Scientific Project of Sichuan Health Commission (grant no. 19ZD005) and the Research and Development Program of The Affiliated Hospital of North Sichuan Medical College (grant no. 2021JC009).

\section{Availability of data and materials}

The datasets used and/or analyzed during the current study are available from the corresponding author on reasonable request.

\section{Authors' contributions}

PL, JW and XR conceived and designed the study. YZ, YX, SL, ZY and HT performed the experiments and data analysis. PL, JW and XR wrote the manuscript. XCT and GJZ analyzed and interpreted the data and revised the manuscript. All authors read and approved the final manuscript. GJZ and XCT confirmed the authenticity of all the raw data.

\section{Ethics approval and consent to participate}

The present study patient experimental protocol was approved by the Ethics Committee of The Affiliated Hospital of North Sichuan Medical College [approval no. 2021ER(A)005]. The processing of clinical tissue samples was performed in strict compliance with the ethical standards of the Declaration of Helsinki. All patients provided written informed consent prior to participation. The animal experimental protocol was approved by The Experimental Animal Ethics Committee of North Sichuan Medical College (approval no. 201927).

\section{Patient consent for publication}

Not applicable.

\section{Competing interests}

The authors declare that they have no competing interests.

\section{References}

1. Siegel RL, Miller KD, Goding Sauer A, Fedewa SA, Butterly LF, Anderson JC, Cercek A, Smith RA and Jemal A: Colorectal cancer statistics, 2020. CA Cancer J Clin 70: 145-164, 2020.

2. Zhu M, Zhang W, Ma J, Dai Y, Zhang Q, Liu Q, Yang B and Li G: MicroRNA-139-5p regulates chronic inflammation by suppressing nuclear factor- $\kappa \mathrm{B}$ activity to inhibit cell proliferation and invasion in colorectal cancer. Exp Ther Med 18: 4049-4057, 2019.

3. Eberlein A, Takasuga A, Setoguchi K, Pfuhl R, Flisikowski K, Fries R, Klopp N, Fürbass R, Weikard R and Kühn C: Dissection of genetic factors modulating fetal growth in cattle indicates a substantial role of the non-SMC condensin I complex, subunit G (NCAPG) gene. Genetics 183: 951-964, 2009.

4. Palou R, Dhanaraman T, Marrakchi R, Pascariu M, Tyers M and D'Amours D: Condensin ATPase motifs contribute differentially to the maintenance of chromosome morphology and genome stability. PLoS Biol 16: e2003980, 2018.

5. Jiang L, Ren L, Chen H, Pan J, Zhang Z, Kuang X, Chen X, Bao W, Lin $\mathrm{C}$, Zhou Z, et al: NCAPG confers trastuzumab resistance via activating SRC/STAT3 signaling pathway in HER2-positive breast cancer. Cell Death Dis 11: 547, 2020.

6. Sun DP, Lin CC, Hung ST, Kuang YY, Hseu YC, Fang CL and Lin KY: Aberrant expression of NCAPG is associated with prognosis and progression of gastric cancer. Cancer Manag Res 12: 7837-7846, 2020

7. Zhang X, Wang H, Han Y, Zhu M, Song Z, Zhan D and Jia J: NCAPG induces cell proliferation in cardia adenocarcinoma via PI3K/AKT signaling pathway. Onco Targets Ther 13: 11315-11326, 2020.

8. Arai T, Okato A, Yamada Y, Sugawara S, Kurozumi A, Kojima S, Yamazaki K, Naya Y, Ichikawa T and Seki N: Regulation of NCAPG by miR-99a-3p (passenger strand) inhibits cancer cell aggressiveness and is involved in CRPC. Cancer Med 7: 1988-2002, 2018

9. Gong C, Ai J, Fan Y, Gao J, Liu W, Feng Q, Liao W and Wu L: NCAPG promotes the proliferation of hepatocellular carcinoma through PI3K/AKT signaling. Onco Targets Ther 12: 8537-8552, 2019.

10. Xu T, Dong M, Wang Z, Li H and Li X: Elevated mRNA expression levels of NCAPG are associated with poor prognosis in ovarian cancer. Cancer Manag Res 12: 5773-5786, 2020.

11. Xue Y, Li PD, Tang XM, Yan ZH, Xia SS, Tian HP, Liu ZL, Zhou T, Tang XG and Zhang GJ: Cytochrome $\mathrm{C}$ oxidase assembly factor 1 homolog predicts poor prognosis and promotes cell proliferation in colorectal cancer by regulating PI3K/AKT signaling. Onco Targets Ther 13: 11505-11516, 2020.

12. Bartel DP: MicroRNAs: Genomics, biogenesis, mechanism, and function. Cell 116: 281-297, 2004.

13. Lee YS and Dutta A: MicroRNAs in cancer. Annu Rev Pathol 4: 199-227, 2009.

14. Grossi I, Salvi A, Baiocchi G, Portolani N and De Petro G: Functional role of microRNA-23b-3p in cancer biology. Microrna 7: 156-166, 2018.

15. Xian Z, Hu B, Wang T, Zeng J, Cai J, Zou Q and Zhu P: lncRNA UCA1 contributes to 5-fluorouracil resistance of colorectal cancer cells through miR-23b-3p/ZNF281 axis. Onco Targets Ther 13: 7571-7583, 2020.

16. Livak KJ and Schmittgen TD: Analysis of relative gene expression data using real-time quantitative PCR and the 2(-Delta Delta C(T)) method. Methods 25: 402-408, 2001.

17. Chen DL, Wang DS, Wu WJ, Zeng ZL, Luo HY, Qiu MZ, Ren C, Zhang DS, Wang ZQ, Wang FH, et al: Overexpression of paxillin induced by miR-137 suppression promotes tumor progression and metastasis in colorectal cancer. Carcinogenesis 34: 803-811, 2013.

18. Zhang G, Zhou H, Xiao H, Li Y and Zhou T: MiR-378 is an independent prognostic factor and inhibits cell growth and invasion in colorectal cancer. BMC Cancer 14: 109, 2014.

19. LoRusso PM: Inhibition of the PI3K/AKT/mTOR pathway in solid tumors. J Clin Oncol 34: 3803-3815, 2016.

20. Ai J, Gong C, Wu J, Gao J, Liu W, Liao W and Wu L: MicroRNA-181c suppresses growth and metastasis of hepatocellular carcinoma by modulating NCAPG. Cancer Manag Res 11: 3455-3467, 2019.

21. Narayanankutty A: PI3K/Akt/ mTOR pathway as a therapeutic target for colorectal cancer: A review of preclinical and clinical evidence. Curr Drug Targets 20: 1217-1226, 2019.

22. Aoki M and Fujishita T: Oncogenic roles of the PI3K/AKT/mTOR axis. Curr Top Microbiol Immunol 407: 153-189, 2017. 
23. Danielsen SA, Eide PW, Nesbakken A, Guren T, Leithe E and Lothe RA: Portrait of the PI3K/AKT pathway in colorectal cancer. Biochim Biophys Acta 1855: 104-121, 2015.

24. Rupaimoole R and Slack FJ: MicroRNA therapeutics: Towards a new era for the management of cancer and other diseases. Nat Rev Drug Discov 16: 203-222, 2017.

25. Campos-Viguri GE, Peralta-Zaragoza O, Jiménez-Wences $H$, Longinos-González AE, Castañón-Sánchez CA, Ramírez-Carrillo M, Camarillo CL, Castañeda-Saucedo E, Jiménez-López MA, Martínez-Carrillo DN and Fernández-Tilapa G: MiR-23b-3p reduces the proliferation, migration and invasion of cervical cancer cell lines via the reduction of c-Met expression. Sci Rep 10: 3256, 2020.

26. Begum S, Hayashi M, Ogawa T, Jabboure FJ, Brait M, Izumchenko E, Tabak S, Ahrendt SA, Westra WH, Koch W, et al: An integrated genome-wide approach to discover deregulated microRNAs in non-small cell lung cancer: Clinical significance of miR-23b-3p deregulation. Sci Rep 5: 13236, 2015.

27. Zhou C, Chen Y, He X, Zheng Z and Xue D: Functional implication of exosomal miR-217 and miR-23b-3p in the progression of prostate cancer. Onco Targets Ther 13: 11595-11606, 2020.
28. Zaman MS, Thamminana S, Shahryari V, Chiyomaru T, Deng G, Saini S, Majid S, Fukuhara S, Chang I, Arora S, et al: Inhibition of PTEN gene expression by oncogenic miR-23b-3p in renal cancer. PLoS One 7: e50203, 2012.

29. Wang J, Xue H, Zhu Z, Gao J, Zhao M and Ma Z: Expression of serum exosomal miR-23b-3p in non-small cell lung cancer and its diagnostic efficacy. Oncol Lett 20: 30, 2020.

30. Zhang Y, Chen D, Zhang G, Wu X, Zhou L, Lin Y, Ding J, An F and Zhan Q: MicroRNA-23b-3p promotes pancreatic cancer cell tumorigenesis and metastasis via the JAK/PI3K and Akt/NF- $\mathrm{KB}$ signaling pathways. Oncol Lett 20: 160, 2020.

31. Chen D, Wu X, Xia M, Wu F, Ding J, Jiao Y, Zhan Q and An F: Upregulated exosomic miR-23b-3p plays regulatory roles in the progression of pancreatic cancer. Oncol Rep 38: 2182-2188, 2017. Attribution-NonCommercial-NoDerivatives 4.0 International (CC BY-NC-ND 4.0) License. 